\title{
Neutrophilic dermatosis of the dorsal hands: response to dapsone monotherapy*
}

\author{
Felipe Siqueira Ramos¹, Flávia Regina Ferreira², Fátima Maria de Oliveira Rabay², \\ Marcia Lanzoni de Alvarenga Lira ${ }^{1}$
}

DOI: http:/ / dx.doi.org/10.1590/abd1806-4841.20187488

\begin{abstract}
Neutrophilic dermatosis of the dorsal hands is considered a rare and localized variant of Sweet's syndrome. Although the etiology is unknown, there are reports of association with infections, neoplasias, autoimmune diseases and medications. Histopathology shows a dense neutrophilic inflammatory infiltrate in the dermis. Treatment is based on the administration of systemic corticosteroids; however, a combination of medications is useful, given the frequency of relapses. The authors report a classic and clinically exuberant case of neutrophilic dermatosis of the dorsal hands, with excellent response to oral dapsone treatment, and offer a brief literature review.
\end{abstract}

Keywords: Dapsone; Hand dermatoses; Sweet syndrome; Vasculitis

\section{INTRODUCTION}

Neutrophilic dermatoses represent a set of entities whose characteristic is the presence of an inflammatory infiltrate rich in neutrophils, without evidence of infectious agents, that can appear in localized cutaneous, generalized cutaneous and systemic forms. ${ }^{1-3}$ Neutrophilic dermatosis of the dorsal hands (NDDH) presents morphological and histopathological characteristics resembling those of Sweet's syndrome (SS) and is considered a rare and localized variant of this syndrome. ${ }^{1,3,4}$

The objective of this report is to present a classic and clinically exuberant case of NDDH that responded excellently to oral dapsone treatment, to highlight the rarity of this entity, and to offer a brief literature review.

\section{CASE REPORT}

A 64-year-old female patient, phototype IV, homemaker, sought the Dermatology Service complaining of "wound on the hand for 40 days". On dermatological examination, she presented with an erythematoviolaceous annular plaque, with a vegetative and exulcerated border, on the dorsum of the right hand (Figure 1). She noted its appearance after visiting a kennel. She brought with her a complete hemogram without significant changes and a culture of the secretion of the lesion, revealing growth of Staphylococcus saprophyticus and Proteus sp. Systemic amoxicillin and sulfamethoxazole/trimethoprim, as well as the topical fusidic acid cream, were used, with partial improvement of the condition; however, relapse occurred after the medication was discontinued.

\footnotetext{
Received 07 August 2017.

Accepted 20 November 2017.

* Work performed at the Hospital Universitário de Taubaté, Universidade de Taubaté, Taubaté (SP), Brasil.

Financial support: None.

Conflict of interest: None.

Dermatology Unit, Hospital Universitário de Taubaté, Universidade de Taubaté, Taubaté (SP), Brazil.

Discipline of Dermatology, Department of Medicine, Universidade de Taubaté, Taubaté (SP), Brazil.
}

MAILING ADDRESS:

Felipe Siqueira Ramos

E-mail: siqueira_ramos@hotmail.com 


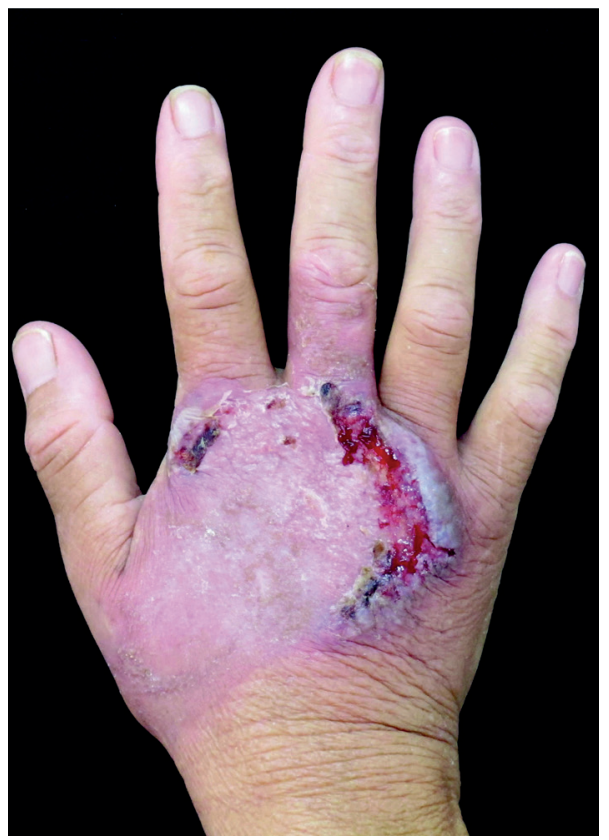

Figure 1:

Dorsum of the right hand. Erythematoviolaceous annular plaque with exulcerated border
In terms of comorbidities, the patient reported having hypertension and diabetes, and was using losartan and metformin. An incisional biopsy of the lesion was conducted, and the anatomopathological examination showed epidermis with parakeratosis, spongiform pustules, and acanthosis; the dermis showed mild edema and a dense, diffuse and predominantly neutrophilic infiltrate, without signs of vasculitis (Figures 2 and 3). Cultures for fungi, bacteria and mycobacteria were negative. Considering the patient's clinical presentation, the results of the anatomopathological exam, and the negative cultures, the diagnosis of NDDH was established. Dapsone therapy was administered at a dose of $100 \mathrm{mg}$ per day. One month later, the patient presented with a nearly complete regression of the lesion (Figure 4).

\section{DISCUSSION}

Described by Robert Douglas Sweet in 1964 as acute febrile neutrophilic dermatosis, SS is characterized by fever, leukocytosis, painful and erythematous plaques preferentially located on the face and extremities, and diffuse neutrophilic infiltrate in the dermis. ${ }^{3,5}$

NDDH was first described by Strutton et al. in 1995, considering six cases of women with lesions resembling SS, but located predominantly on the dorsum of hands. As the histopathological findings showed associated leukocytoclastic vasculitis, the entity was denominated pustular vasculitis of the hands. ${ }^{6}$ Later, in 2000, Galaria et al. described three cases having clinical characteristics resembling the previously described pustular vasculitis of the hands, as well as dense neutrophilic dermal infiltrate, but without evidence of vasculitis. ${ }^{7}$ Therefore, they proposed to rename the entity as NDDH, a variant of SS. ${ }^{7}$

Currently, NDDH is a rare entity, with about 60 cases described in the literature. ${ }^{1}$ It preferentially affects female patients with an average age of 62 , commonly manifesting as papules, plaques,

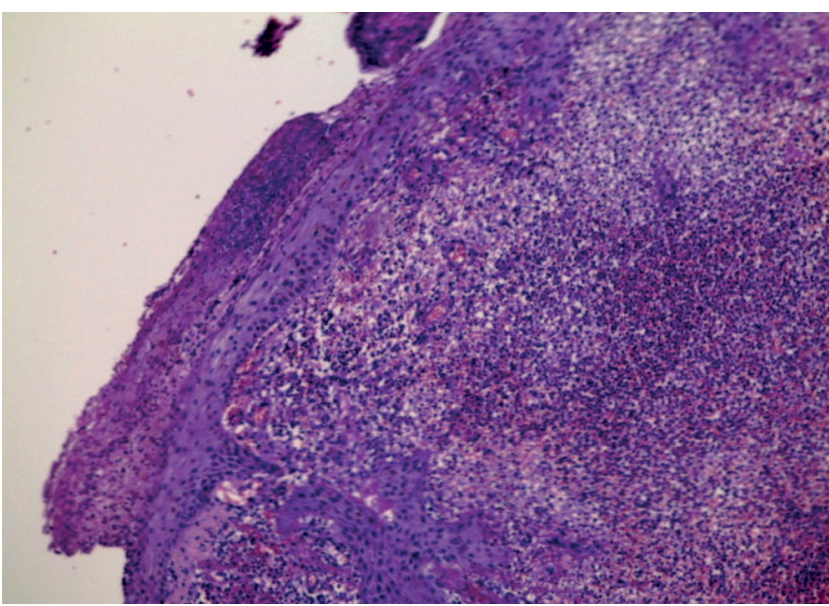

FIGURE 2: Skin with solid and diffuse inflammatory infiltrate in the dermis (Hematoxylin \& eosin, $x 40$ )

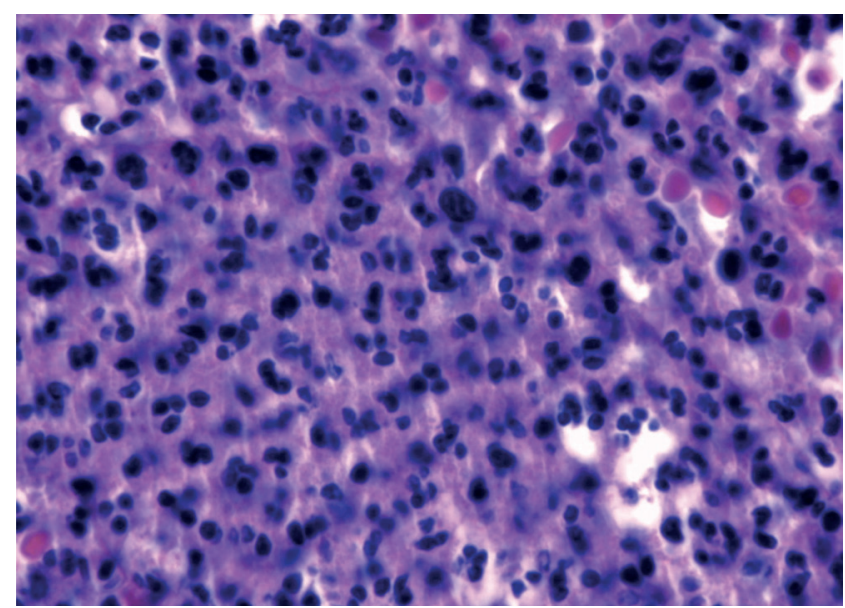

FIGURE 3: The infiltrate is predominantly composed of neutrophils, some with leukocytoclasia (Hematoxylin \& eosin, x400)

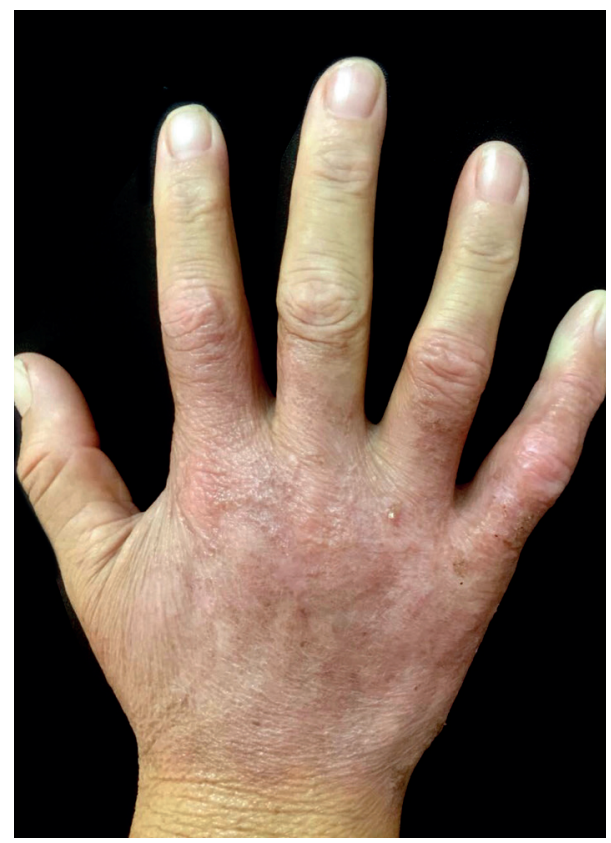

Figure 4:

Dorsum of the right hand. Nearly complete regression. Residual erythema 
pustules, blisters and ulcerations, and usually limited to the dorsum of one or both hands ${ }^{1}-$ a similar situation was observed in this case. Signs of vasculitis can be observed, as previously indicated. In this patient, such signs were not present. Malone et al. considered that the presence of vasculitis in some cases was probably a secondary event, related to the intensity of the neutrophilic inflammatory infiltrate and to the duration of the lesions' evolution, not representing true vasculitis. ${ }^{8}$

Similar to what occurs in other neutrophilic dermatoses, the cutaneous lesions of NDDH may be accompanied by systemic manifestations - most frequently fever, general unwellness, arthralgia, or peripheral adenopathy-none of which were observed in this patient. ${ }^{1}$

The etiology of NDDH is unknown, though there are reports of its association with infections of the respiratory tract, hepatitis $\mathrm{C}$ infection, inflammatory bowel disease, neoplasms (particularly hematological), autoimmune diseases (seropositive arthritis), medications (lenalidomide, thalidomide), vaccines and exposure to fertilizers. ${ }^{1,3,5}$ None of these associations have been observed in the patient thus far.

The differential diagnoses of this entity include pyodermas, other neutrophilic dermatoses, and erythema elevatum diutinum. ${ }^{1,5}$

The treatment is based on the administration of systemic corticosteroids. However, given the frequency of relapse, the combination of dapsone and colchicine is useful, and they also serve as corticosteroid-sparing agents. Methotrexate, cyclosporin, azathioprine, indomethacin, minocycline, tetracycline, danazol, clofazimine, and potassium iodide are used less frequently. ${ }^{1,9,10}$ Tacrolimus and topical corticosteroids can be used on small lesions with good results. ${ }^{1,10}$ In this report, the patient used only oral dapsone and presented nearly complete regression of the lesion after one month of treatment.

This classic and exuberant presentation, and the excellent response to the use of oral dapsone, motivated us to report this case and highlight the importance of knowledge about this entity and its possible associations, which is essential for its correct and complete management. $\square$

\section{REFERENCES}

1. Macias VC, Labareda J, Themido R, Viana I. Dermatose neutrofílica do dorso das mãos - a propósito de dois casos clínicos. Revista SPDV. 2013;71:71-6.

2. Duquia RP, Almeida HL Jr, Vettorato G, Souza PR, Schwartz J. Neutrophilic dermatosis of the dorsal of the hands: Acral sweet syndrome? Int J Dermatol. 2006:45:51-2.

3. Bonamigo RR, Razera F, Olm GS. Neutrophilic dermatoses: part I. An Bras Dermatol. 2011:86:11-25.

4. Imaoka K, Kaneko S, Harada Y, Ota M, Furumura M, Morita E. Neutrophilic dermatosis of the palms. J Dermatol. 2012;39:949-51.

5. Kaur S, Gupta D, Garg B, Sood N. Neutrophilic dermatosis of dorsal hands. Indian Dermatol Online J. 2015:6:42-5.
6. Strutton G, Weedon D, Robertson I. Pustular vasculitis of the hands. J Am Acad Dermatol. 1995;32:192-8

7. Galaria NA, Junkins-Hopkins JM, Kligman D, James WD. Neutrophilic dermatosis on the dorsal hands: pustular vasculitis revisited. J Am Acad Dermatol. 2000;43:870-4.

8. Malone JC, Slone SP, Wills-Frank LA, Fearneyhough PK, Lear SC, Goldsmith LJ, et al. Vascular in $\square$ ammation (vasculitis) in Sweet syndrome. A clinicopathologic study of 28 biopsy specimens from 21 patients. Arch Dermatol. 2002;138:345-9.

9. DiCaudo DJ, Connolly SM. Neutrophilic dermatosis (pustular vasculitis) of the dorsal hands: a report of 7 cases and review of the literature. Arch Dermatol. 2002;138:361-5

10. Del Pozo J, Sacristán F, Martínez W, Paradela S, Fernández-Jorge B, Fonseca E. Neutrophilic dermatosis of the hands: presentation of eight cases and review of the literature. J Dermatol. 2007;34:243-7.
AUTHORS'CONTRIBUTIONS

\section{Felipe Siqueira Ramos}

iD ORCID 0000-0002-3109-4359

Approval of the final version of the manuscript, Design and planning of the study, Preparation and writing of the manuscript, Collecting, analysis and interpretation of data, Effective participation in research orientation, Intellectual participation in propaedeutic and/or therapeutic conduct of studied cases, Critical review of the literature, Critical review of the manuscript

Flávia Regina Ferreira

D ORCID 0000-0001-5679-4282

Approval of the final version of the manuscript, Design and planning of the study, Preparation and writing of the manuscript, Collecting, analysis and interpretation of data, Effective participation in research orientation, Intellectual participation in propaedeutic and/or therapeutic conduct of studied cases, Critical review of the literature, Critical review of the manuscript

How to cite this article: Ramos FS, Ferreira FR, Rabay FMO, Alvarenga Lira ML. Neutrophilic dermatosis of the dorsal hands: response to dapsone monotherapy. An Bras Dermatol. 2018;93(5): 730-2. 